\title{
Effective Community Mobilization: Lessons Learnt from Implementing the Integrated Care Model in Zimbabwe
}

\author{
M. Mhlanga, M. Chidzonga, and C. Haruzivishe
}

\section{ABSTRACT}

Globally, there has been a heated debate on the effectiveness of community mobilization in improving maternal and child health outcomes. Sustainable Development Goal number three aims at attaining Universal Health Coverage to improve health outcomes to the most marginalized and vulnerable populations. This paper outlines the key lessons that we learnt from the implementation of a novel community mobilization model, the Integrated Care Model (ICM) in Zimbabwe. The keys lessons were:

- To determine effect of community models, set parameters of measuring the effect at protocol development.

- Effective community mobilization in maternal and child health needs saturation coverage.

- Meaningful community engagement is critical throughout the mobilization cycle.

- Community Health Workers derive motivation from different things that are cheap and cost-effective.

- Community models should be integrated into the mainstream health delivery system for sustainability.

- Community models should be integrated into the mainstream health delivery system for sustainability.

- Participatory facilitation approaches yield better results that lectures in community health promotion.

- Community Health Workers should have comprehensive kits that are continually replenished for effectiveness in early diagnosis and treatment of childhood illnesses

We conclude that integration of the ICM model into the main health delivery system ensures the consistent transference of correct knowledge and skills for better maternal and child health. The Government of Zimbabwe and other developing countries should consider implementing such an approach in their respective community health strategies in order to reduce preventable child morbidity and mortality.

Keywords: Child Morbidity; Integrated Care Model.
Published Online: October 5, 2020

ISSN: $2593-8339$

DOI: 10.24018 /ejmed.2020.2.5.483

\section{Mhlanga*}

University of Zimbabwe, Faculty of Medicine and Health Sciences, Harare, Zimbabwe.

(e-mail: profmaxmhlanga7@gmail.com)

M. Chidzonga

University of Zimbabwe, Faculty of Medicine and Health Sciences, Harare, Zimbabwe.

(e-mail: mtmchidzonga@yahoo.com)

C. Haruzivishe

University of Zimbabwe, Faculty of Medicine and Health Sciences, Harare, Zimbabwe

(e-mail: claraopha@ gmail.com)

*Corresponding Author

\section{INTRODUCTION}

Globally, there is currently much controversy over the effectiveness of the community mobilization models in reducing child morbidity. A number of models have been adduced on both sides of the question, but very few studies address the question: What is the effectiveness of community intervention approaches in reducing child morbidity in lowresource settings? [1]

Effective community mobilization approaches are those that effectively utilize community health workers to maintain consistent and regular contact with households, developing strong partnerships with the served communities to reduce child morbidity. Currently there is still limited evidence about the effectiveness of integrated community- based approaches in reducing child morbidity. Policy makers in health are lured by community programmes that are both sustainable and effective. Close integration between community health interventions and primary health facilities are critical elements of effectiveness [2].

In this paper, we present lessons learnt for the implementation of an Integrated Care model to improve maternal and child care outcomes through increasing use of preventive and responsive care practices in targeted communities in Mashonaland East Zimbabwe. Figure 1, below is a schematic presentation of the Integrated Care Model (ICM) represented as the intervention and the conventional community mobilization model (to the left), represented as the control intervention. 


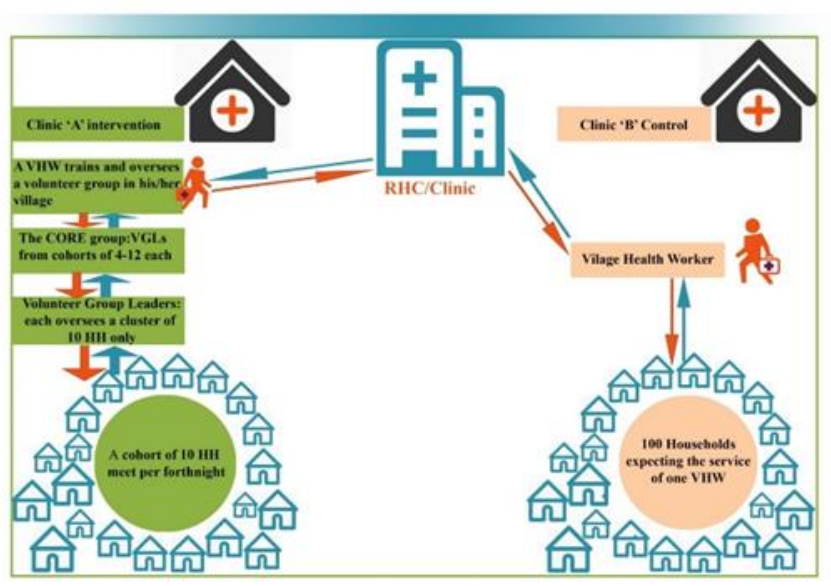

Fig. 1. The Integrated Care Model compared with the conventional community care model.

\section{LESSONS LEARNT}

Lesson 1: To determine effect of community models, set parameters of measuring the effect at protocol development

Many researchers who have endeavored to measure the effect and impact of community mobilization models have had serious challenges in determine the impact of their model when compared with other models or conventional Interventions. Our experiences in this study, have proved that it is critical for public health programmers who intend to measure effect through clinical trials to be able to specify how they will measure intervention dosage. It is critical to answer the following questions before commencing your study:

1. How are we going to measure intervention dosage by study arm?

2. How are we going to measure intervention intensity by study arm?

3. How will we measure intervention specificity by study arm?

Each study should clearly specify the definition of these key study components on the context of the study, so that meaningful analysis can be carried out at the end of study.

Lesson 2: Effective community mobilization needs saturation coverage

The greatest barrier to effective community health systems strengthening in low to medium income countries has been the unavailability of skilled and trained community health workers who are fully who can easily reach every household with life-saving maternal and child health care messages and services. These community health workers should also be able to monitor key practices and health care behaviours to save lives of mothers and children in their catchment area. Saturation coverage at household level addresses knowledge gaps, myths and misconceptions, harmful cultural and religious practices and power dynamics at household level.

Reach out to all households had a multiplier effect in interpersonal behaviour change communication and door-todoor health promotion (through home visits) based on culturally appropriate interaction around relevant evidence had a significant positive impact on health practices.

When compared with the conventional community health care model, the Integrated Care Model, yielded better results owing to the level of immersion that the later model had. With a community health worker within the reach of 10 households, it was easier to monitor behaviour and practices and referrals were made earlier resulting in better prognosis for mothers and children who got it or need health care services. Saturation coverage will foster collective ownership and accountability on health matters by all households leading to greater uptake of life-saving interventions such as the Expanded Programme of Immunisation, PMTCT among others MNCH services. The ICM model results in a statistically significant effect on the uptake of preventive and responsive practices that prevent maternal and child illnesses, complications and preventable deaths among women of child bearing age in the study intervention arm.

Lesson 3: Meaningful community engagement is critical throughout the mobilization cycle

Our experiences have proven that communities sit down and observe, usually with a critical mind, those programmes and projects that have been imposed on them, with their little or no involvement. This has been the biggest challenge to the sustainability of many projects beyond their lifespan.

Adoption of new practices and approaches at community level, do not happen overnight and take a lot of patience and active engagement of all stakeholders, especially those who house power in different domains such as the traditional leadership, religious leaders, the sage, opinion leaders, traditional healers and other influential members who are in the health delivery structure.

The model improved positive health-seeking behaviours on $\mathrm{MNCH}$ at a low programming cost. Social barriers were broken through the engagement of influential leaders and custodians of culture thereby promoting positive health practices at low cost for mothers and their children. The "scale-free social network" was harnessed in this approach thereby strengthening the social cohesion through engaging community-derived resources and mobilizing large numbers of women and men as volunteers who facilitated peer-to-peer behaviour change.

Engaging health workers from the onset builds capacities, effectively improves dialogue within the health system and the performance of frontline health workers. It was interesting to learn that when Village Health Workers are not fully engaged, they themselves could be the ones to shoot down a public health programme that is meant to ease their work and improve health outcomes due to poor dynamics. Yet at the same time, when each new community health programme amplifies their voice and acknowledge them, putting them in the centre of the community health programmes, the results are bountiful and sustainable.

In designing policies and frameworks for community health, policy makers should consider using both the bottomup and top-to-bottom approach or use collaborative approaches in engaging communities on their health matters. This fosters ownership and collective accountability, since it empowers communities, enabling them to have a say in issues relating to their own health.

Lesson 4: Community Health Workers derive motivation from different things that are cheap and cost-effective

Motivation issues remain a bone of contention in community mobilization especially where voluntarism is concerned. Every individual does a cost-benefit analysis at individual level and introspects to determine whether there is anything for them to benefit in participating in a given 
community health program. While monetary incentives have been acknowledged as good incentives that can motivate community health workers to conduct their routine primary health care activities in their catchment areas, they have had a challenge of sustainability beyond the lifespan of a project. In Zimbabwe there are more than 78 different types of community cadres who have enrolled or recruited for different health programmes and some of their roles died away as soon as the project ended because, the government could not sustain the monetary incentives in as much as they would require their services. For interventions that have a lasting impact, we recommend that, the community and the government develop local systems that can sustain the gains and continue to motivate the community volunteers through small tokens of appreciation which could include monetary incentives.

We have observed that in Zimbabwe and some other developing countries, the World Bank is supporting the Results Based financing programme to promote the attainment of maternal and child health indicators and sustainable development goals. Health centres receive funds for them to subside MNCH health services cost and promote uptake of services by mothers and children. The health centres that took advice to spare some funds they received for good performance to support community health workers through small incentives had far much more sustainable health outcomes compared to those who prioritized facility based services only. Such small initiatives can be sustained if the community is contionuolsy appraise of the impact this is having on maternal and child morbidity and mortality.

Our model was effective in promoting task shifting and the decentralization of power. Community volunteers were motivated and incentivized by the community recognition and respect they got from villagers. The valued skills they possessed and used to save lives in their respective villages and households also motivated them to do more. Programme impact and community support also sustains community volunteers more than material things such as money could. In order to keep them energized and motivated, and to prevent fatigue and maintain the momentum, it will be prudent to rotate the community volunteers after every two years. The study intervention did not only reduce child morbidity but also increased volunteer retention.

Lesson 5: Communities should be involved in the maternal and child health outcome monitoring

We learnt that communities are keen to know how their villages are performing compared to others in their catchment are on maternal and child health care uptake, practices and other outcomes such as maternal and child morbidity and mortality.

It is very critical that the data collected from the communities monthly or quarterly be processed and analysed, using simple pictorials, graphs, pie charts, line graphs to illustrate how they are performing on programmes like the child immunisation programme, Antenatal care, deliveries and many others. Working with the community leaders there should be a regular feedback mechanism, where the results are communicated and interpreted and communities, compare themselves against themselves (horizontal trend analysis) over different periods of time. Communities are also able to compare themselves against other communities in their catchment area or beyond. Communities always have an inert drive to excel and be better than their neighbors and such energy can be harnessed to achieve better health outcomes.

We have seen such a practice becoming a good stimuli for look and learn (exchange visits) as communities seek to be the best leading to the perpetuation of best practices in maternal and child care. Establishing a community health monitoring and evaluation system that monitors health indicators at household level improve community health programming in many dimensions. Our experiences, revealed that head count statistics generated easily through such a community system improved efficiency and effectiveness of community programs like the malaria control programs such as the Indoor Residual Spraying Program and the Long Lasting Insecticidal Nets (LLINs) distribution programme. In our experience, the coverage rate as of such programmes and other out-reach programmes were consistently above $95 \%$ and always surpassed those of the health centres where the conventional mobilization system was used. The Integrated Community Model effectively increased precision and promoted timeous reporting on maternal and child health indicators through effective home visits.

Lesson 6: Community models should be integrated into the mainstream health delivery system for sustainability

Integrating this mobilization approach into the mainstream health delivery system ensured that transference of knowledge and skills necessary for effective and lasting community health development is continuous even after the research was concluded. This will reduce costs and prevent the duplication of resources and efforts for the same cause and provides a sustainable and effective way of delivering quality health care at community level. The fragmented approach that is currently used in most countries is costly and pays fewer dividends. A fragmented approach to community health care delivery has been the reason behind weak community health delivery system, since life-saving activities have only been effective only during the periods they are funded and effectively monitored by external staff and experts.

Nowadays, governments in low-to medium income countries are look for cost-effective community health solutions that are pragmatic and that have demonstrated that they easily fit into conventional system. Local health centre staff in primary health care centres, are more likely going to be committed in supporting community health initiatives that fit in the way of doing business as opposed to stand alone programmes. Integrated systems amplify the effect of initiatives as they are also likely going to complement and strengthen existing initiatives.

Community models that integrated in the conventional health delivery system, have a strong potential to outlast funding and project cycles. Such models can also achieve sustainable impact by focusing on changing the mindset and attitudinal components of the community as a whole on maternal and child health care.

Lesson 7: Participatory facilitation approaches yield better results that lectures in community health promotion

In Zimbabwe, Village Health Workers use opportune moments and events to raise health awareness in places like funerals or in village meetings that the kraal head or headmen would have called and they are usually given 10 to 20 minutes 
to talk about any health matters they may deem necessary hence they use the 'supermarket approach' to raise awareness and conduct health education. This means that most of the health education sessions are not really planned and they are ad hoc. On the same note, the Village Health Workers use the 'supermarket approach' to facilitate health promotion sessions. A 'supermarket approach' implies that they try to provide all information on maternal and child in one lecture say of 10 minutes to promote utilization of services. This approach has proved to be ineffective in community health promotion as it fails to bring attention to details.

For example, on childhood illnesses, instead of focusing on one diseases condition, its prevalence, causes, prevention, management and let's danger signs, the Village Health Worker would just brush through and at the end the knowledge and skills that the care-givers adopt from the session are very minimal and ineffective for adoption of optimal maternal and child care practices.

In our intervention model, we adopted the participatory approaches for facilitation of health education sessions. The volunteers at community level had facilitation manuals that had sessions which were systematically arranged and followed the life cycle approach. There were sessions on maternal health during pregnancy, during labour and delivery, post-natal care, care of the newborn, care of infants, prevention of childhood illnesses, danger signs and importance of seeking health care services early among other key topics.

The sessions were facilitated using the 'learning circle approach'. This is a scenario where mothers/care-givers sit in a circle including the facilitator. This removed power-barriers and allowed all participants to participate as equals and this fostered full attention of each member as there was eyecontact between all members. It was easier for the community facilitators in these circles to validate acceptance of new information or resistance through direct observation of nonverbal cues such as facial expressions. The community groups should be self-propagating, as they set their own bylaws, deliberate on what they want to learn and how, decide their own succession plans and rotation cycles for new leaders to avoid burn-out and fatigue.

The manual employed participatory methods such as the use of stories with a gap, testimonials, simulations, picture books, songs and role play to address maternal and child health care issues. Understanding the current practices and the rationale behind them was very useful in debunking negative norms and cultural practices on childcare. The caregivers would learn from each other challenging each other until a consensus is reached. The role of the facilitator was to enhance the positive messages after the discussions. The mixed beliefs, perceptions and practices on maternal and child care, coupled with the women's eagerness to learn new things sustained their availability and participation in the educational sessions in their respective villages.

Women composed interesting and innovative songs and dramas that brought out clearly the recommended take home messages and unveiled the hidden practices that were context-specific which had negative consequences to maternal and child health. The groups served as vehicles for behaviour modification and were also effective in promoting peer-to-peer education and behaviour monitoring.
Community women are not motivated to adopt certain behaviours when they feel that these have been imposed by external agents through a top-down approach. However, if they are given time to conceptualize behaviour and own it, there is an increased likelihood that they will adopt the behaviour and sustain it. The study revealed that social learning is the outcome for both health promoters and the local people which results in mutual understanding and better quality of life.

Lesson 8: Community Health Workers should have comprehensive kits that are continually replenished

One of the biggest challenges that the Village Health Workers raised as a deterrent factor to their effective functionality in the communities they serve, was the absence of key life-saving drugs (Amoxicillin, anti-malaria drugs), and screening tools such as scales, thermometers, MUAC tapes, testing kits and other ancillaries for early detection of illnesses and management. Governments in developing countries should ensure that community health workers are fully equipped with kits that allow them to treat childhood illness at very early stages. Since early treatment is cheaper than referral, community health workers should be fully capacitated and effectively monitored in their screening and management of childhood illnesses. All community health workers should have community health manuals that they use continuously to refer to for consistency in information disseminated and for them to also cover more content on the Integrated Management of Newborn and Childhood illnesses.

\section{CONCLUSION}

Given our experience in the implementation of an integrated community model to improve maternal and child health care practices and outcomes, we conclude that integration of such a model into the main health delivery system will ensure the consistent transference of correct knowledge and skills for better maternal and child health. This happens through the perpetuation of a cycle of continuous health education, monitoring and promotion. Such an integrated approach will improve both maternal and child health outcomes by reducing the incidence of maternal and child morbidity. The Government of Zimbabwe and other developing countries should consider implementing such an approach in their respective community health strategies in order to reduce preventable child morbidity and mortality.

\section{REFERENCES}

[1] Perry, H., Morrow, M., Borger, S., Weiss, J., DeCoster, M., Davis, T. 2015 Care Groups I: an innovative community-based strategy for improving maternal, neonatal, and child health in resource-constrained settings. Glob Health Sci Pract. 3(3):358-36.

[2] World Relief. 2015 Innovating for child health: An evaluation of an integrated care group model in Rwanda. Final report. United States Agents of International Development. 1-12. 